\title{
Genetic Invention of Fast and Optimal Broad-band Stokes/Mueller Polarimeter Designs
}

\author{
Paul Anton Letnes \\ paul.anton.letnes@gmail.com \\ Pål Gunnar Ellingsen \\ paalge@gmail.com \\ Ingar Stian Nerbø \\ nerbo@ntnu.no \\ Department of Physics
Norwegian University of
Science and Technology
N-7491 Trondheim, Norway
}

\author{
Lars Martin Sandvik Aas \\ lars.martin.aas@gmail.com \\ Morten Kildemo \\ morten.kildemo@phys.ntnu.no
}

\begin{abstract}
We have applied a genetic algorithm to generate optimal polarimeter designs for a selected wavelength interval, assuming known dispersion relations of the components. Our results are improvements on previous patented designs based on ferroelectric liquid crystals.
\end{abstract}

\section{Categories and Subject Descriptors}

J.2 [Physical Sciences and Engineering]: Physics; G.1.6 [Optimization]: Global Optimization

\section{General Terms}

Design

\section{Keywords}

Polarimetry, Ellipsometry, Optical design, Genetic algorithms

\section{INTRODUCTION}

Vision is perhaps the most important sense we have, and light is widely used for measurement and detection in science and technology. Techniques based on light usually have the advantages of being non-destructive and non-invasive, and have the possibility of performing measurements at high speed and large (even astronomical) distances. Unfortunately, the human eye and many measurement techniques are only able to detect the intensity of light. As light consists of oscillating electric and magnetic fields, it is a vectorial quantity. The orientation properties of these fields is referred to as the polarization of the light. A polarimeter is an instrument that measures the polarization of light to gain additional information. Such instruments are applied in a wide range of fields: from astronomy to characterization of semiconductor components, remote sensing, and medical diagnostics.

Any polarization state of light can be described by a 4 element Stokes vector, S [2]. A Stokes polarimeter measure these elements by using optical components such as a linear polarizer and e.g. ferroelectric liquid crystals (FLC) to measure the intensity of light projected along different

Copyright is held by the author/owner(s).

GECCO'11, July 12-16, 2011, Dublin, Ireland.

ACM 978-1-4503-0690-4/11/07. polarization states. For dispersive optical components, optimal projection states can only be achieved for a single wavelength. Finding the optimal polarimeter operating over a broad spectrum of wavelengths turns out to be a challenging optimization problem, well suited for genetic algorithms (GA).

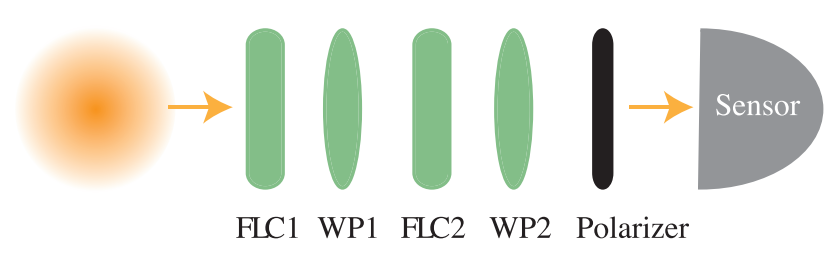

Figure 1: An example 2-FLC polarimeter where the polarization state of incident light is analyzed by the Polarization State Analyzer and a light intensity detector.

The change of a polarization state can be described by a $4 \times 4$ transformation matrix called a Mueller matrix, connecting an incoming Stokes vector to an outgoing Stokes vector, $\mathbf{S}_{\text {out }}=\mathbf{M S}_{\mathrm{in}}$. The Mueller matrix can describe the effect of any linear interaction of light with a sample or an optical element. Polarization effects contained in a Mueller matrix could be diattenuation, retardance, and depolarization. Due to dispersion the Mueller matrix will in general be wavelength dependent.

The measured intensities of a polarimetric measurement are arranged into a vector $\mathbf{b}$, and is related to the unknown Stokes vector $\mathbf{S}$ through $\mathbf{b}=\mathbf{A S}$, where $\mathbf{A}$ is a system matrix, which can be found from the Mueller matrices of the components. The unknown Stokes vector can then be found by inverting $\mathbf{A}, \mathbf{S}=\mathbf{A}^{-1} \mathbf{b}$. The noise in the intensity measurements $\mathbf{b}$ will be amplified by the condition number $(\kappa)$ of $\mathbf{A}$ in the inversion. Therefore $\kappa$ should be as small as possible [4]. The condition number of $\mathbf{A}$ is given as $\kappa=\|\mathbf{A}\|\left\|\mathbf{A}^{-1}\right\|$, which for the 2-norm equals the ratio of the largest to the smallest singular value. The best condition number that can be achieved for a polarimeter is $\kappa=\sqrt{3}$ [4]. If more than 4 measurements are performed, $\mathbf{A}$ will not be square, and the Moore-Penrose pseudoinverse is used to invert $\mathbf{A}$.

To evaluate the performance of a polarimeter design, we compare the inverse condition number to the theoretically 
optimal value $(1 / \sqrt{3})$. We define an fitness function $(f)$ as

$$
\frac{1}{f}=\frac{1}{N_{\lambda}} \sum_{n=1}^{N_{\lambda}}\left(\kappa^{-1}\left(\lambda_{n}\right)-1 / \sqrt{3}\right)^{4} .
$$

We take $\left(\kappa^{-1}-1 / \sqrt{3}\right)$ to the 4 th power to punish unwanted peaks in $\kappa$ more severely.

While the GA is able to handle any kind of optical components, we focus on systems based on FLCs as polarization modulators, with fixed waveplates "sandwiched" between them to increase the condition number. The design we set out to find was one based on three FLC retarders and three fixed waveplates. Each FLC has two variables, namely its thickness and its orientation angle. The same is true for the fixed waveplates. This yields a 12-dimensional search space: six components with two variables each.

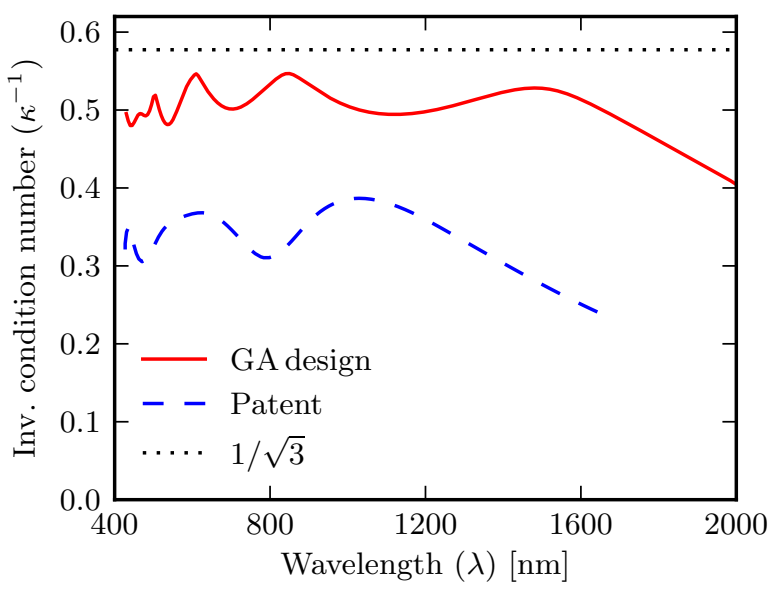

Figure 2: Inverse condition number $\left(\kappa^{-1}(\lambda)\right)$ for a GA-generated and a previously patented design [1].

In our GA we represent polarimeter designs using a traditional binary genome. Each component is assigned a number of bits for the orientation angle and a number of bits for its thickness. Given these parameters the condition number $\kappa$ of the system matrix $A$ can be determined [3].

The genetic operator that was used for mutation was the simple bit-flip operator. The mutation rate per individual was typically set to 0.2 per generation. Crossover was performed by standard multi-point crossover. Experience indicates that two crossover points combined with a crossover rate of 0.7 gives the best convergence performance. The selection protocol we used was tournament selection with $K=4$ individuals in the tournament pool and $\varepsilon=0.3$ probability of an "underdog" selection. The elitism rate was set to 1 individual per generation. A population of 500 individuals evolving over 600 generations was used. Several equivalent simulation runs were performed with different initializations of the random number generator.

\section{RESULTS}

The best polarimeter design found by GA is shown in Figure 2. For comparison with previous designs, we also show a recently patented design. Both designs are based on three FLCs. The new design is useful over a broader spectral range and has lower noise amplification due to a lower condition number. The condition number is improved

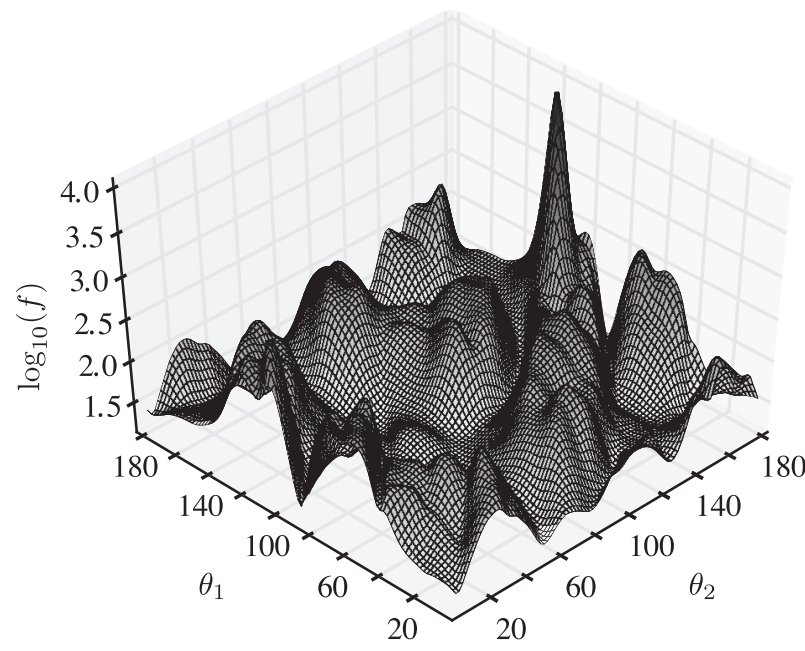

Figure 3: Fitness landscape around the optimal value for the GA-generated design. $\theta_{1}$ and $\theta_{2}$ are the orientation angles of two of the components.

by up to a factor of 2 , which means the noise amplification can be reduced by a factor of 2 .

One can get an impression of how complex the fitness landscape is from Figure 3, where a plot of $f$ depending on 2 of the 12 parameters are shown.

In conclusion, the use of GA allows for quick generation of good polarimeter designs. The generated designs outperform previously published and patented designs.

\section{ACKNOWLEDGEMENTS}

We thank prof. Keith Downing at the Dept. of Computer and Information Science at NTNU for helpful discussions.

\section{REFERENCES}

[1] D. Cattelan, E. Garcia-Caurel, A. de Martino, and B. Drevillon. Device and method for taking spectroscopic polarimetric measurements in the visible and near-infrared ranges. Patent application, 2937732(France), 2010.

[2] E. Collett. Polarized light. Fundamentals and applications. Marcel Dekker, Inc., New York, 1993.

[3] P. A. Letnes, I. S. Nerbø, L. M. S. Aas, P. G. Ellingsen, and M. Kildemo. Fast and optimal broad-band Stokes/Mueller polarimeter design by the use of a genetic algorithm. Opt. Express, 18(22):23095-23103, 2010 .

[4] J. S. Tyo. Noise equalization in Stokes parameter images obtained by use of variable-retardance polarimeters. Opt. Lett., 25:1198-1200, 2000. 